\title{
Study on the Microstructure of the Pinctada martensii Pearls and Its Significance
}

\author{
Wenlong Wu, ${ }^{1}$ Liping Zhang, ${ }^{1,2}$ Zhijun Yang, ${ }^{1,2}$ and Weisheng Hou ${ }^{1,2}$ \\ ${ }^{1}$ Department of Earth Science, Sun Yat-Sen University, Guangzhou 510275, China \\ ${ }^{2}$ Guangdong Provincial Key Laboratory of Mineral Resource Exploration \& Geological Processes, Guangzhou 510275, China
}

Correspondence should be addressed to Zhijun Yang, yzjdoctor@163.com

Received 1 March 2012; Accepted 8 May 2012

Academic Editors: J. Casado, M. Mączka, and Y. Ueno

Copyright ( $) 2012$ Wenlong Wu et al. This is an open access article distributed under the Creative Commons Attribution License, which permits unrestricted use, distribution, and reproduction in any medium, provided the original work is properly cited.

\begin{abstract}
The results of a microscopy, SEM-EDS, XRD, FTIR, and Raman spectra study of the nacres of the Pinctada martensii pearls from Zhanjiang city, China shows that they can be classified as the high-quality, medium-quality, and inferior-quality pearls. Aragonite, the main inorganic mineralogy in the nacres, was crystallized and grown up in the compartments formed by the silk and radial organic sheets originating from organic matters secreted by the mantle of mollusks. The crystalline orientations of aragonite tablets were changed from the (002), (012) and (102) crystalline plane nets in the early to the (002) crystalline plane net only in the later. The formation processes of the microstructure of the nacres could be divided into three stages. In the early stage, the precursor particles of aragonite nucleated and grew up fast; then, porous aragonite aggregates consisting of the fine aragonite crystals were formed. In the middle stage, the aragonite crystals directionally grew up to form the aragonite tablets and microlayers. The surface of the aragonite tablets and microlayers are rough and few porous, and the edges of the crystals were serrated. In the last stage, the aragonite tablets in the aragonite microlayer mixed perfectly together to form high-quality aragonite layer whose surface was smooth and perfect.
\end{abstract}

\section{Introduction}

The nacres of pearl, products of the biomineralization of the mollusks, are natural nano-organic-inorganic composite materials. Their special physical and chemical properties have excited more and more attentions of material scientists, mineralogists, and biologists. For instance, the nacres of pearl are widely used in the production of the pearl wool and pearl fiber because their break resistance performance can reach about 3000 times of that of the pure inorganic materials [1]. The special physical and chemical properties of the nacres of pearl depend on their unique microstructure whose formation process are typical self-assembly process of nanomaterials. Additionally, the degree of order of aragonite crystals in the nacres highly impacts the quality of the pearl. Currently, based on the above reasons, the research of the microstructure of the nacres of pearl has become an international hotspot and frontier subject. Up to now, to explain the formation mechanism of the microstructure of the nacres of pearl, the compartment theory [2], the bridge theory $[3,4]$, and the template theory $[5,6]$ have been established. Although the established modes are effective on the studies of the configuration and formation process of microstructural of the nacres and their application, it is very difficult to explain the formation of the crystal preferred orientation, crystalline polymorphy, and crystalline domains [7] in the nacres of pearl.

As we know, the nacres are formed by the interaction of aragonite tablets and the organic matters [8]. Generally, in the nacres there are $>95 \% \mathrm{wt}$ calcium carbonate in inorganic matters which are mainly composed of aragonites [9]. Aragonites can be aggregated to form aragonite tablets (the size is about $0.4 \sim 2 \mu \mathrm{m}$, the diameter is about $3 \sim 10 \mu \mathrm{m}$ ), and aragonite microlayers in the next. During a long period of time, the aragonite tablets had been believed to be directional arrangement and their C-crystal axis was only vertical to the stratification plane of the nacres [10]. Namely, the crystalline orientation of aragonite tablets is unique because they were crystallized along with the crystalline plane nets which were matching with the formation periods 
of the organic matters. However, the results of recent studies indicated that the aragonite tablets caused the diversity characteristic crystalline orientation and their C-crystal axis was not necessarily perpendicular to the stratification plane of the nacres [7, 11]. In addition, using the established modes of the formation of the nacres, it is difficult to explain the existence and formation of the aragonite crystallized domains which result in the excellent mechanical property of the nacres [12]. Therefore, the study of the microstructure and the formation mechanism of the nacres is still a subject worth exploring and it will provide new scientific proofs for the formation of the nacres and new ideas for the research and production new materials.

In this paper, we focus on the nacres of Pinctada martensii pearls, characterize their microtopography, microcomposition, and microstructure by using an optical microscope, scanning electron microscope (SEM), energy dispersive spectrometer (EDS), powder X-ray diffraction (XRD), fourier transform infrared spectra (FTIR), and Raman spectra (Raman), and discuss their significances to the formation mechanism of microstructure of the nacres.

\section{Sample and Experiment}

The Pinctada martensii pearl samples were collected from the Dajin countryside, Zhanjiang city, Guangdong province which is one of the most important production bases of seawater pearls in China.

Based on the pearl luster, size, and surface flaw ratio, the samples can be classified as the high-quality pearls, medium-quality pearls, and inferior-quality pearls. Some of the samples were cut out two parallel polishing planes for the micromorphology observation and micro-Raman experiments. Some of the nacres were manually separated and grinded into fine powders with size of 200 meshes for XRD and FTIR experiments.

An optical microscope and a scanning electronic microscope (SEM, S-520/ISIS-300 type) with energy dispersive spectroscopy (EDS) whose element range of detection was ${ }^{11} \mathrm{Na} \sim{ }^{92} \mathrm{U}$ and minimum weight of element detection was $0.5 \%$ wt were used to characterize the crystal habit, the microtopographic, and the microcomponents. Before the SEM-EDS experiments, the samples were pretreated by gold spraying.

The powder X-ray diffraction experiments were carried out at the D-MAX 2200VPC type X-ray diffraction instrument which adopted $\mathrm{Cu} K \alpha(\lambda=1.5405 \AA)$ as excitation ray and used carbon filter. In the experiment, the diffraction pattern is $\theta \sim 2 \theta$ coupled progressive scan, the scan angle range is $2 \theta=3^{\circ} \sim 66^{\circ}$, and the scan speed is $10^{\circ} / \mathrm{min}$. The other parameters in the experiment were $U$ (voltage) $=$ $40 \mathrm{kV}$ and $I$ (electronic current) $=30 \mathrm{~mA}$. The JADE software package was used to analyze the X-ray diffraction spectra.

The FTIR absorption spectra were recorded on the powder samples which are pressed into tablets with potassium bromide $(\mathrm{KBr})$ from $400 \sim 4000 \mathrm{~cm}^{-1}$ with $4 \mathrm{~cm}^{-1}$ resolution and 6 times scan at room temperature in transmission mode using a Bruker Equinox-55 FTIR spectrometer coupled with an infrared microscope. The OriginPro 7.5 software package was used to analyze the FTIR spectra.

A Renishaw InVia Confocal Micro-Raman spectrometer was employed for the Micro-Raman experiment, using excitation by the $514.5 \mathrm{~nm}$ line of $\mathrm{an} \mathrm{Ar}^{+}$laser. Micro-Raman spectra were recorded from $50 \sim 4000 \mathrm{~cm}^{-1}$ with $1 \mathrm{~cm}^{-1}$ resolution, from 4 points at the same intervals, and from the inside to outside of the nacres.

\section{Results}

3.1. Optical Microscopy. Figure 1 shows the optical stereophotographs of the high-quality pearls (Figures 1(a) and $1(\mathrm{~b})$ ), medium-quality pearls (Figures $1(\mathrm{c})$ and $1(\mathrm{~d})$ ) and inferior-quality pearls (Figures 1(e), 1(f), and 1(g)).

In the three types of the pearls, the inner part of them was pearl nucleus and the outer part of them was the nacres (Figure 1). The average thickness of the nacres of highquality pearls was 0.375 millimeter, the average thickness of the nacres of medium-quality pearls was 0.75 millimeter, and the average thickness of the nacres of inferior-quality pearls was 0.90 millimeter. Uniform color, smooth stratification plane, close contact with pearl nucleus, and dense structure in the nacres of the high-quality pearls indicated that they experienced an excellent formation process (Figures 1(a) and $1(\mathrm{~b}))$. Between the nacres and pearl nucleus of the medium-quality pears, many gaps which were filled by black materials resulted in loose structure and low toughness of the nacres (Figures 1(c) and 1(d)). Non uniform color, uneven stratification plane, and loose contact with pearl nucleus, many gaps which were filled by black or golden materials, and some spherulitic and needle cylinder crystals in the nacres of the inferior-quality suggest that they experienced a fast formation process (Figures $1(\mathrm{e}), 1(\mathrm{f})$, and $1(\mathrm{~g})$ ).

3.2. Scanning Electronic Microscopy. Figure 2 shows the secondary electronic images of the high-quality pearls (Figures 2(a), 2(b), and 2(c)), medium-quality pearls (Figures 2(d), $2(\mathrm{e})$, and $2(\mathrm{f}))$, and inferior-quality pearls (Figures 2(g), $2(\mathrm{~h})$, and $2(\mathrm{i}))$.

The nacres of the high-quality pearls had obvious lamellar structure which consisted of many crystalline microlayers whose thickness was about $1.2 \mu \mathrm{m}$ and width was $1.86 \mu \mathrm{m}$ and edge line was zigzag (Figures 2(a), 2(b), and 2(c)). On the crystalline microlayers, there were some polygon aragonite sheets on which some crystals were forming (see the arrow in the Figure 2(a)). Figure 2(b) showed the coexistence of some tough and smooth crystalline planes which were, respectively, related to the crystallizing materials and the integrating aragonite sheets. Figure 2(c) showed a typically brick wall structure of the nacres with the organic as syrup and the inorganic as wall.

The nacres of the high-quality pearls also had obvious lamellar structure and there are some growing aragonite sheets whose thickness was about $0.25 \mu \mathrm{m}$ and width was $1.25 \mu \mathrm{m}$ (Figure 2(d)). The big aragonite sheets and holes in the nacres of medium-quality pearls (Figure $2(\mathrm{~d})$ ) implied the aragonites experienced a fast-growing process and were aggregating. The connection of the two dome-like parts 


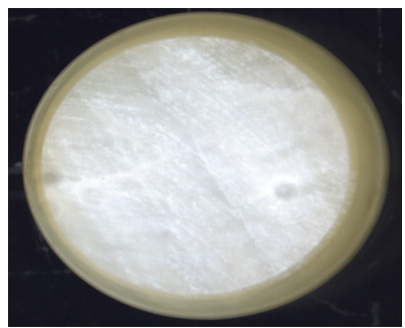

$2 \mathrm{~mm}$

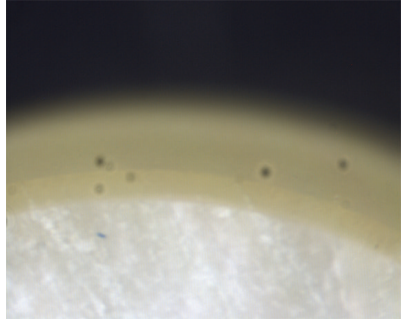

$1 \mathrm{~mm}$

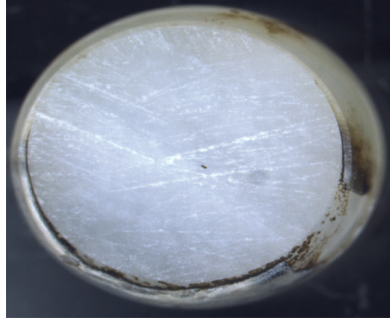

$2 \mathrm{~mm}$

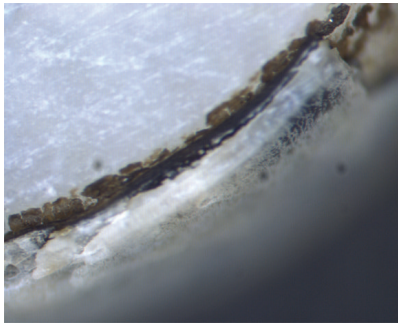

$\overline{1 \mathrm{~mm}}$

(a)

(b)

(c)

(d)

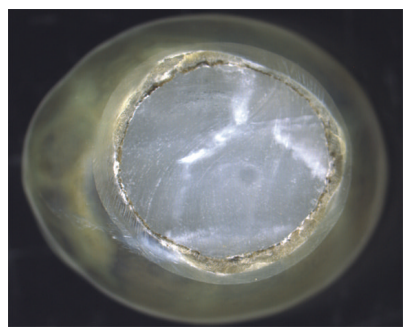

$2 \mathrm{~mm}$

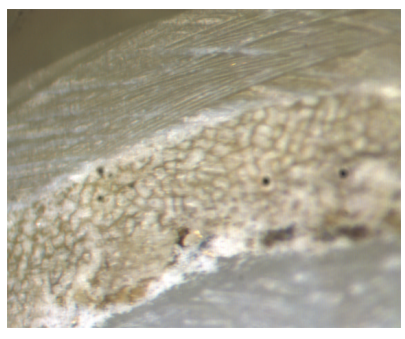

$1 \mathrm{~mm}$

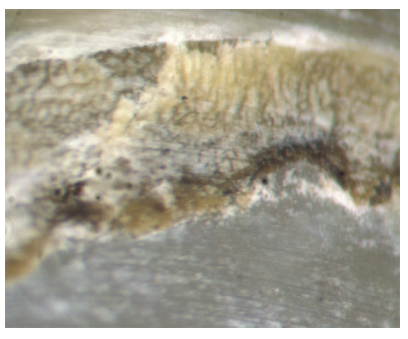

$1 \mathrm{~mm}$

(e)

(f)

(g)

Figure 1: Stereophotographs of the different-quality Pinctada martensii pearls. (a)-(b) high-quality pearls; (c)-(d) medium-quality pearls; (e)-(f) inferior-quality pearls.

of the crystalline layers (Figure 2(f)) indicated that the microlayers of the nacres tend to integrate.

The junction between the nacre and the nucleus of inferior-quality pearls also showed a lamellar structure (Figure $2(\mathrm{~g})$ ). Figure $2(\mathrm{~h})$ showed there were some fine crystalline particles and block crystalline aggregates on the microlayers and many columnar or lanciform crystals among the microlayers of the nacres which indicated the preferential orientation vertical to the stratification plane of the nacres and might be the so-called mineral bridges [4]. Figure 2(i) showed there were some fibrous organic matters on the aragonite sheets and in the integration locations among the aragonite sheets. Meanwhile, there were some forming crystals enclosed by radial organic matters (see the white arrow in Figure 2(i)) in the nacres which indicated the formation of inorganic crystals were close related to the organic matters and were limited by the compartment formed by the radial organic matters.

3.3. Energy Dispersive Spectroscopy. Figure 3 showed the energy dispersive spectroscopy (EDS) of the nacres of the inferior-quality pearls. The energy dispersive spectra of Figures 3(a) and 3(b) were, respectively, gotten from black arrow positions in Figures 2(h) and 2(i).

The Figure 3(a) showed that the block crystals mainly contained $\mathrm{Ca}$ element, but contained a little $\mathrm{Fe}$ and $\mathrm{Al}$ elements (the gold element should be excluded because the samples were sprayed by gold). The Figure 3(b) showed that the crystals enclosed by radial organic matters mainly contained $\mathrm{Ca}$ element also, but contained a little Fe and K elements. In this scenario, the $\mathrm{Fe}, \mathrm{Al}$, and $\mathrm{K}$ elements might affect the formation of the inorganic crystals in the nacres of the inferior-quality pearls.

3.4. X-Ray Diffraction. Figure 4 shows the XRD diffraction patterns of powder samples of the nacres of high-quality pearls (Figure 4(a)), medium-quality pearls (Figure 4(b)), and inferior-quality pearls (Figure 4(c)), and the standard XRD diffraction patterns of aragonite (Figure 4(d)), and calcite (Figure 4(e)).

According to the XRD diffraction patterns of the nacres of three types of the pearls similar to the standard XRD diffraction patterns of aragonite, aragonites were the main inorganic matters in the nacres of three types of the pearls. However, the weak diffraction peak $\left(2 \theta=29.559^{\circ}\right.$, $d=3.020 \AA$ ) attributed to the (104) crystalline plane net of calcite indicated there were small amount of calcites in the nacres of inferior-quality pearls.

In Figure 4(d), the strongest standard diffraction peak of aragonite is of the (111) crystalline plane nets. But in Figures 4(a), 4(b) and 4(c), the strongest XRD diffraction peak is the (012) crystalline plane net of aragonite whose C-crystal axis is oblique to the stratification plane of the nacres. So in the samples, the (012) crystalline plane net of aragonite has strong preferential orientation. Additionally, comparing the relative intensity $(I \%)$ of diffraction of the samples with the standard relative intensity of aragonite, the present authors found the (002) and (102) crystalline plane nets of aragonite whose C-crystal axis is, respectively, vertical and oblique to the stratification plane of the nacres 


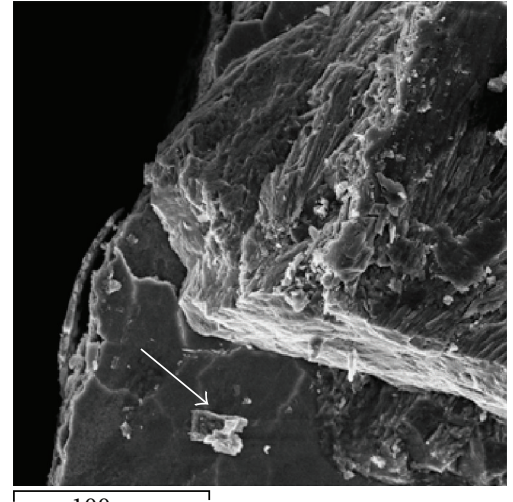

$100 \mu \mathrm{m}$

(a)

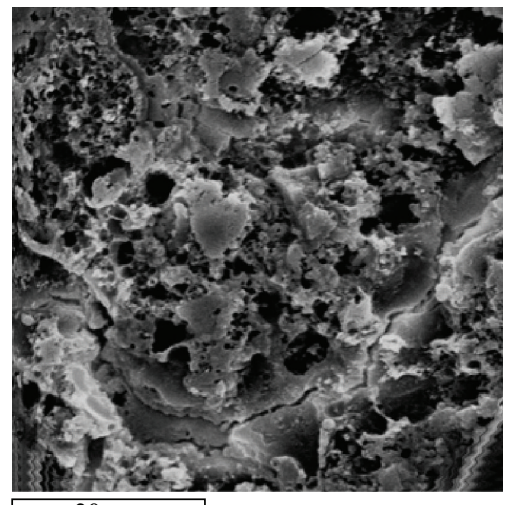

$20 \mu \mathrm{m}$

(d)

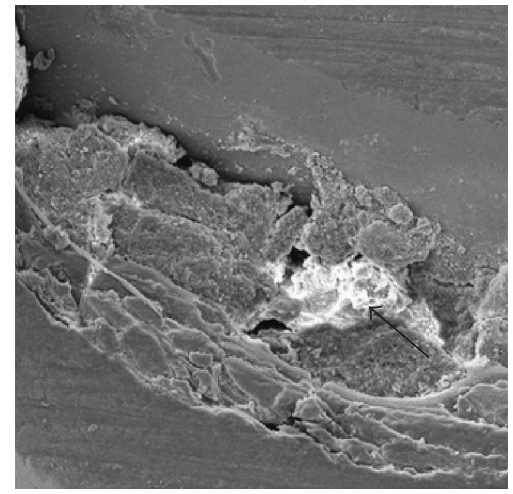

$200 \mu \mathrm{m}$

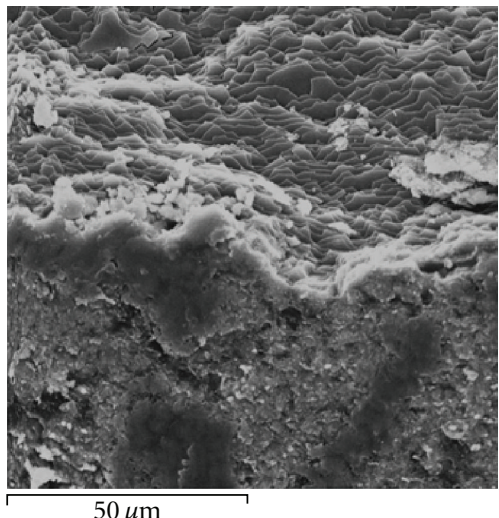

(b)

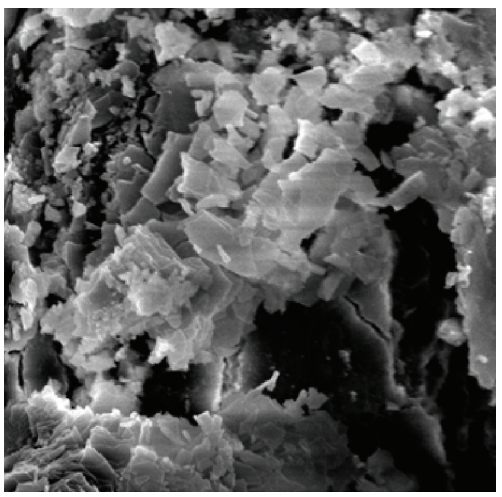

$10 \mu \mathrm{m}$

(e)

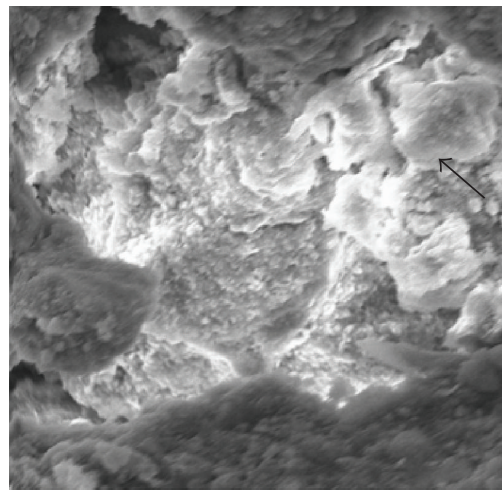

$50 \mu \mathrm{m}$

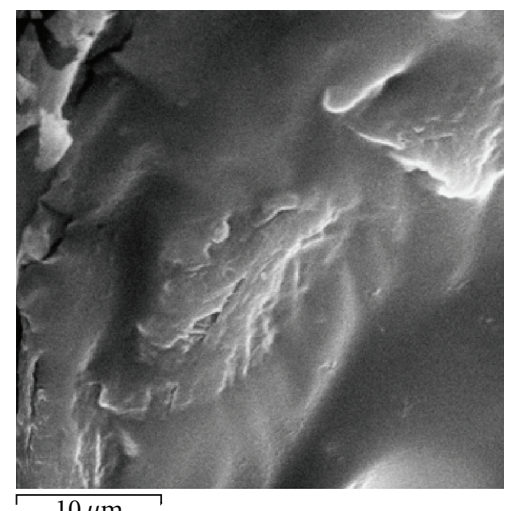

$10 \mu \mathrm{m}$

(c)

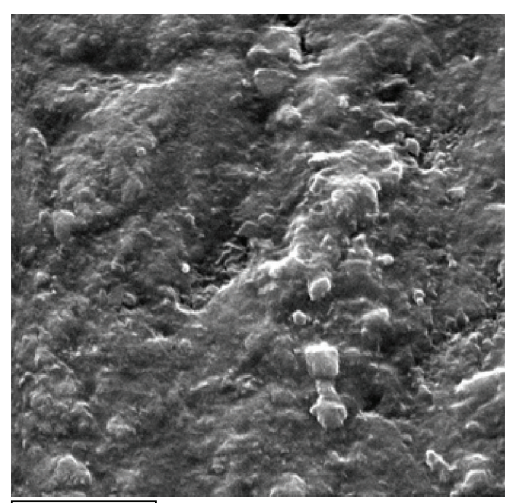

$10 \mu \mathrm{m}$

(f)

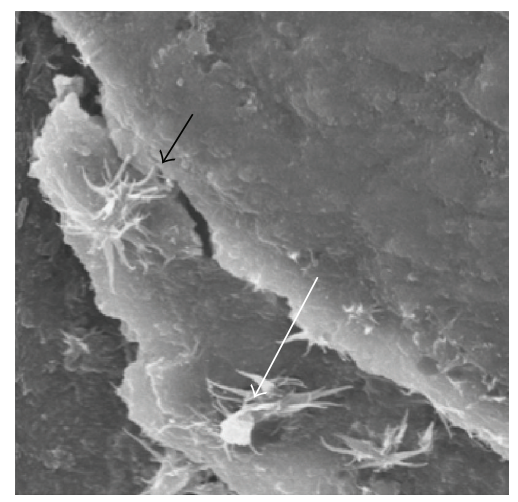

$10 \mu \mathrm{m}$

(g)

(h)

(i)

FIGURE 2: Second electron images of the nacres of the different-quality pearls. (a)-(b) high-quality pearls; (c)-(d) medium-quality pearls; (e)-(f) inferior-quality pearls.

also had strong preferential orientation. For example, in Figures $4(\mathrm{c})$ and $4(\mathrm{~d})$, the relative intensities $(I \%)$ of the (002) diffraction peak $\left(2 \theta=31.239^{\circ}, d=2.861 \AA\right)$ and (102) diffraction peak $\left(2 \theta=36.202^{\circ}, d=2.479 \AA\right)$ are $31.1 \%$ and $36.0 \%$, respectively, which are higher than the relative intensities $(19.8 \%$ and $31.1 \%)$ of the $(002)$ and (102) standard diffraction peaks of aragonite. Therefore, the aragonites have various preferential orientations.
3.5. Fourier Transform Infrared Spectroscopy. Figure 5 shows the FTIR spectra of the nacres of high-quality pearls (Figure 5(a)), medium-quality pearls (Figure 5(b)), and inferior-quality pearls (Figure 5(c)).

The IR spectra of three kinds of pears were similar and could be divided into three bands. Namely, the $700-1786 \mathrm{~cm}^{-1}$ absorption bands related to the Carbonate groups of aragonite, the $2521-3100 \mathrm{~cm}^{-1}$ absorption bands 


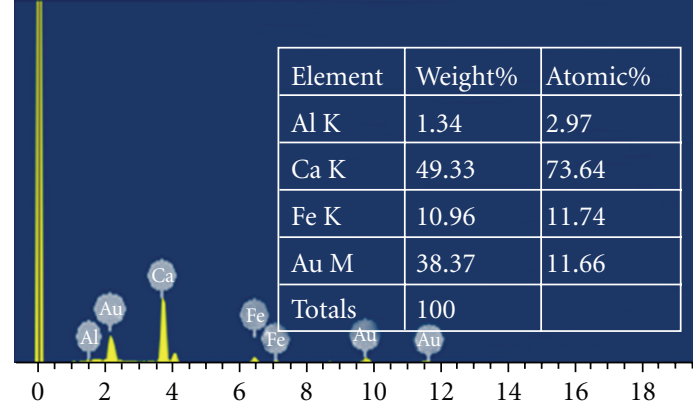

Full scale 2477 cts cursor: $0 \quad(\mathrm{keV})$

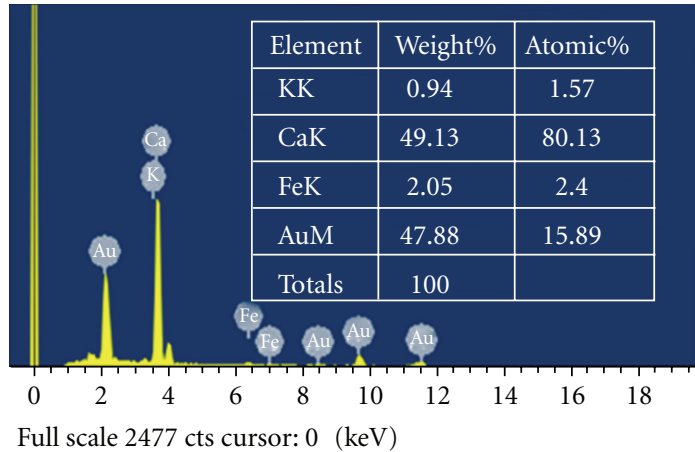

(b)

FIGURE 3: Full scale of energy dispersive spectra of the nacres of the inferior-quality pearls. (a) the arrow in Figure 2(h); (b) the arrow in the Figure 2(i).

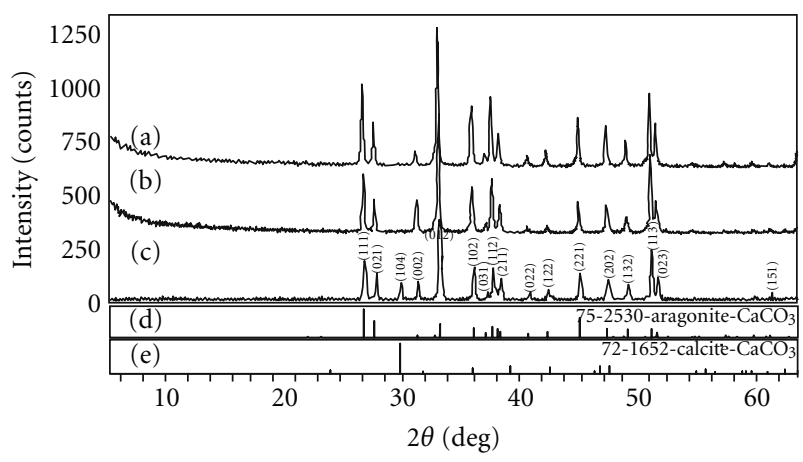

FIGURE 4: X-ray diffraction patterns of the nacres of the differentquality pearls. (a) High-quality pearls; (b) medium-quality pearls; (c) inferior-quality pearls; (d) aragonite crystal; (e) calcite crystal.

related to organic matters, and $\sim 3410 \mathrm{~cm}^{-1}$ absorption peak attributed to water [13].

In the aragonite-related absorption bands, the $\sim 1786 \mathrm{~cm}^{-1}$ absorption peak was attributed to the double degenerate asymmetric stretching vibration of the $\mathrm{CO}_{3}{ }^{2-}$, the $\sim 1082 \mathrm{~cm}^{-1}$ absorption peak was due to the symmetric stretching vibration of the $\mathrm{CO}_{3}{ }^{2-}$, the $\sim 860 \mathrm{~cm}^{-1}$ absorption peak was assigned to the ex-plane bending vibration of $\mathrm{CO}_{3}{ }^{2-}$, and the $700-712 \mathrm{~cm}^{-1}$ absorption peak was attributed to the double degenerate in-plane bending vibration of the $\mathrm{CO}_{3}{ }^{2-}$. Generally, the above four vibration peaks were very sharp and determined exactly. However, due to the overlay of the FTIR peak of the organic matters, the $\sim 1473 \mathrm{~cm}^{-1}$ peak attributed to the double degenerate asymmetric stretching vibration of the $\mathrm{CO}_{3}{ }^{2-}$ was wide and slow and has error in discriminating the aragonite $[14,15]$.

In the organic matters-related absorption bands, the $\sim 2922 \mathrm{~cm}^{-1}$ peaks were due to the asymmetric and symmetric stretching vibration of $\mathrm{sp}^{3}$-hybridized $\mathrm{C}-\mathrm{H}$ bonds, respectively, the $\sim 2521 \mathrm{~cm}^{-1}$ absorption peak was attributed to the organic matters which contained Hydroxyl $(-\mathrm{OH})[13]$.

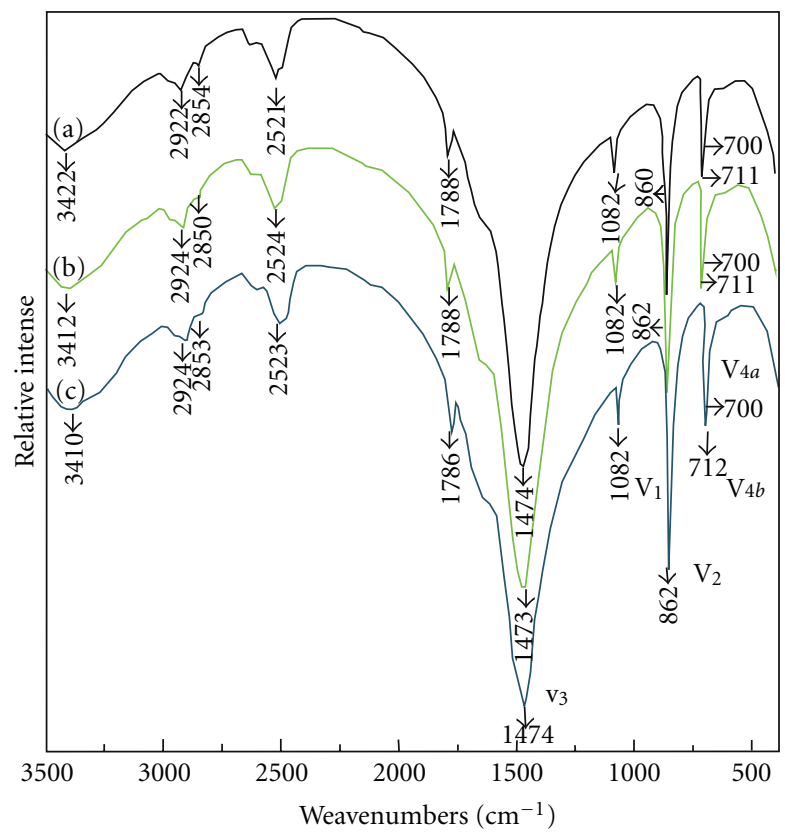

FIGURE 5: Typical FTIR spectra of the nacres of the differentquality pearls (a) High-quality pearls. (b) medium-quality pearls; (c) inferior-quality pearls.

3.6. Raman. Figure 6 shows the Raman spectra from 4 points at the same intervals and from the inside (YZ1, ZZ1 and LZ1, resp.) to outside (YZ4, ZZ4, and LZ4, resp.) of the nacres of high-quality pearls (Figure 6(a)), medium-quality pearls (Figure 6(b)), and inferior-quality pearls (Figure 6(c)).

In Figure 6, $1084 \mathrm{~cm}^{-1}$ peak which is one of the eigen Raman shift of aragonite is very sharp and its intensity increases step by step $(\mathrm{YZ} 1 \rightarrow \mathrm{YZ} 3, \mathrm{ZZ1} \rightarrow \mathrm{ZZ} 3$ and $\mathrm{LZ1} \rightarrow$ LZ3) and only decreases in the outside of the nacres (YZ4, ZZ4, and LZ4), which indicate that the degree of order of aragonite increased and the effect of aragonite increased in the microstructure formation of the nacres. In fact, during the formation of the nacres, the aragonites existing in the organic matters in the form of microparticles initially 


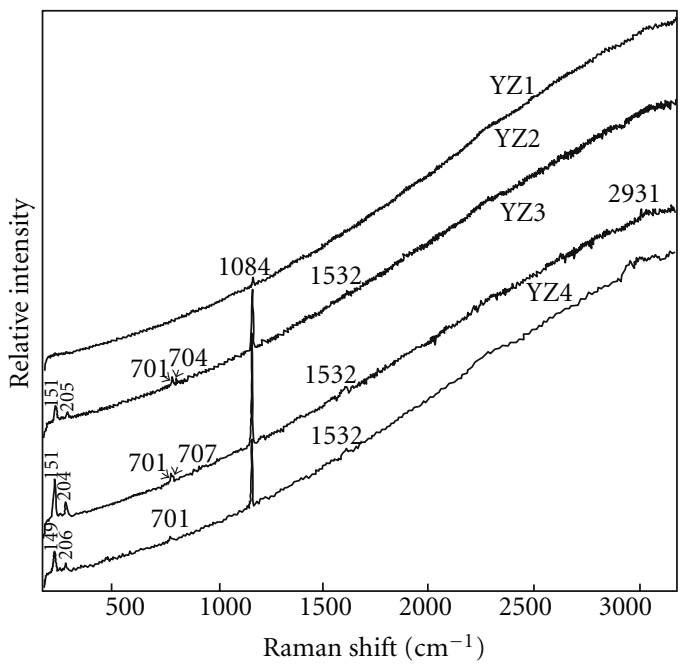

(a)

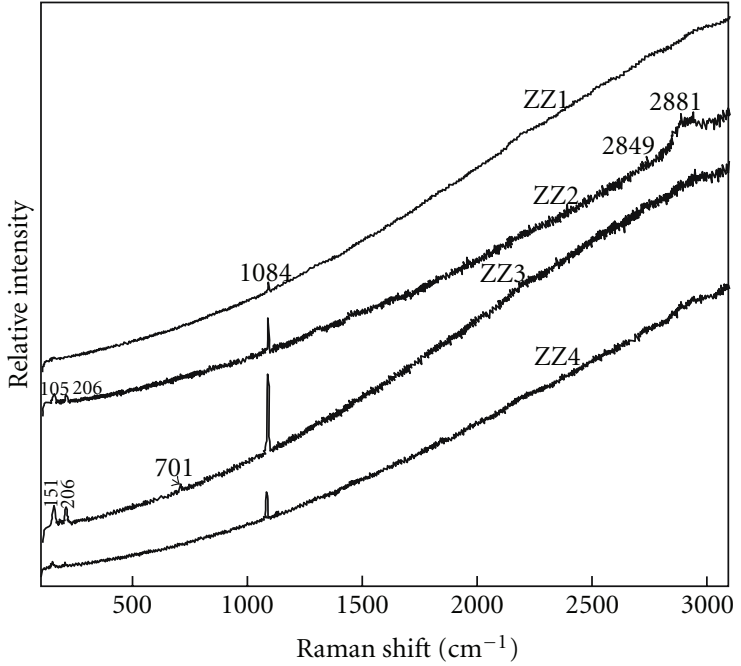

(b)

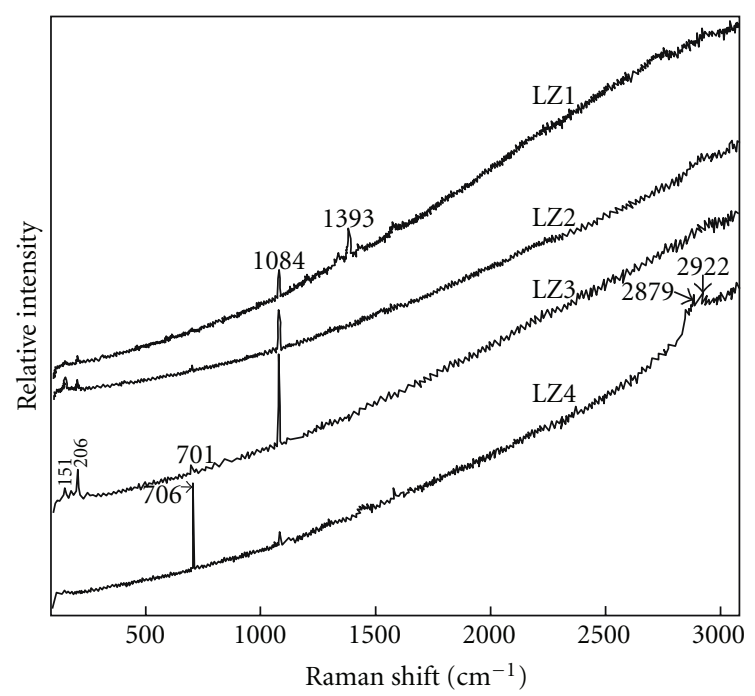

(c)

Figure 6: Typical Raman spectra from the inside to outside of the nacres of the different quality pearls. (a) High-quality pearls; (b) mediumquality pearls; (c) inferior-quality pearls.

fast nucleated and grew. Then the aragonite grew up and optimized its structure and aggregated. In addition, the integral area of $\sim 1084 \mathrm{~cm}^{-1}$ peak of high-quality peals is bigger than of medium-quality pearls than of inferior-quality pearls and the value of full-width half maximum (FWHM) of $\sim 1084 \mathrm{~cm}^{-1}$ peak of high-quality peals is smaller than of medium-quality pearls than of inferior-quality pearls (Table 1), which indicated the quality of pearls was positively correlated to the degree of order of aragonite.

$\sim 701 \mathrm{~cm}^{-1}$ peak which is the other eigen Raman shift of aragonite shows a similar rule as the $1084 \mathrm{~cm}^{-1}$ peak, but it is worth pointing out that the $701 \mathrm{~cm}^{-1}$ peak can be split into the $704 \mathrm{~cm}^{-1}$ and $707 \mathrm{~cm}^{-1}$ two vibration models (see the YZ2, YZ3 in Figure 6(a)). The present authors speculate the key reason is the organic matters inducing the lattice distortion of aragonite.
Besides, the $701 \mathrm{~cm}^{-1}$ peak becomes weak and slow (see the YZ4 in Figure 6(a)) and even disappears (see the ZZ4 in Figure 6(b)), which indicated that there were some low-crystalline aragonite particles on the microlayers. In Figure 6(c), the obviously strong $706 \mathrm{~cm}^{-1}$ peak of the LZ4 which differed from the other two types of pearls indicated that the degree of order of microstructure of the inferiorquality pearls was low.

The $\sim 150 \mathrm{~cm}^{-1}$ and $\sim 206 \mathrm{~cm}^{-1}$ peaks which are the other intrinsic peaks of aragonite [16] become stronger from the inside to the outside of the nacres, which may be related to the effects of the organic matters [17].

In Figure 6(a), there are many peaks attributed to organic matters. For instance, the $1532 \mathrm{~cm}^{-1}$ Raman shift is related to the carotenoid [18] which decides the color of the pearls, the weak $2849 \mathrm{~cm}^{-1}, 2879 \mathrm{~cm}^{-1}, 2881 \mathrm{~cm}^{-1}, 2922 \mathrm{~cm}^{-1}$, 
TABLE 1: Integral area and value of FWHM of $1084 \mathrm{~cm}^{-1}$ Raman shift of the different quality pearls.

\begin{tabular}{lcc}
\hline Samples & $\begin{array}{c}\sim 1084 \mathrm{~cm}^{-1} \\
\text { FWHM } \\
\left(\mathrm{cm}^{-1}\right)\end{array}$ & $\begin{array}{c}\text { Integral area (S1) } \\
(\text { a.u })\end{array}$ \\
\hline High quality pearl (YZ3) & 5.02 & 37647 \\
Medium quality pearls (ZZ3) & 5.05 & 28944 \\
Inferior quality pearls (LZ3) & 5.32 & 24538 \\
\hline
\end{tabular}

and $2931 \mathrm{~cm}^{-1}$ peaks may correspond to organic matters containing $\mathrm{C}-\mathrm{H}$ bond and Hydroxyl [13].

\section{Discussion}

(1) Many strong diffraction peaks of aragonite in Figure 4 and strong vibration peaks of aragonite in Figures 5 and 6 indicated that the aragonite was the main mineral phase of the calcium carbonate in the nacreous layers. Actually, the imbricate microstructure of the nacres was just originated from the interaction between the aragonites and the organic matters. However, due to the changes of crystalline conditions, calcites could be formed from calcium carbonate in the nacres, which might be the key reason that there are weak diffraction peak of calcium carbonate in the XRD diffraction diagram of inferior-quality pearls (Figure 4). Consequently, the present authors thought the small amounts of calcites lowered the quality of pearls and resulted in crystalline polymorphy of calcium carbonate.

(2) Generally, during the biomineralization and formation of the microstructure of the nacres, organic matters control the nucleation and grow-up of the inorganic crystals. At the same time, the inorganic crystal types are related to the facts that calcium element in the calcium carbonate can be substituted by some trace elements [19]. In this scenario, the trace elements $(\mathrm{Fe}, \mathrm{Al}, \mathrm{K}$ ) were responsible for the formation of calcites in the nacres of inferior-quality pearls.

On the one hand, the $\sim 2922 \mathrm{~cm}^{-1}$ and $\sim 2850 \mathrm{~cm}^{-1}$ IR absorption peaks and the $1532 \mathrm{~cm}^{-1}$ Raman shift related to carotenoids implied that there were some organic matters in the nacres. On the other hand, based on the facts that the organic matters extracted from the nacres could effectively induce the nucleation and grow-up of the calcium carbonate and the types and contents of organic matters decided the degree of order of inorganic crystals [19-23], previous research results [24-26] concluded that the organic matters could induced lattice distortion of aragonite and lowered its degree of order and nanometer size effects of aragonite could lowered its degree of order also. In this scenario, comparing the $862 \mathrm{~cm}^{-1}$ IR peak related to aragonite of the samples with the peak of standard inorganic origin aragonite, the obvious blue shift $\left(7.5 \mathrm{~cm}^{-1}\right)$ indicated that there existed complex coordination effects between the organic and inorganic matters. Additionally, in the optical microscope photos of medium-quality pearls (Figures 1(c), and $1(\mathrm{~d})$ ) and inferior-quality pearls (Figures $1(\mathrm{e})$, and $1(\mathrm{~g})$ ), the osteoporosis structure and heterogeneous color of the nacres, the black or grey organic periostracum, and gaps between the periostracum and pearl nucleus also implied the organic matters got variations which might affect the crystallization of aragonites and result in lowering the quality of the pearls.

(3) In Figure 2(i), the bulk crystals enclosed by radial organic matters, which were first observed in the nacres, indicated that the growth of inorganic crystals was controlled by organic matters. In fact, Nakahara et al. [2] thought that the inorganic matters (such as aragonite) were crystallized and grown up in the compartments formed by the silk and radial organic sheets originating from organic matters secreted by the mantle of mollusks [2]. In addition, previous research results $[5,27]$ showed that the proteins could control the crystalline types and orientations of calcium carbonate. For instance, Pif 80 protein could affect the formation of aragonite and resulted in its C-crystal axis orientations vertical to the stratification plane of the nacres [27]. In this scenario, with regard to the formation of aragonite crystals in the nacres, the present authors thought the organic matters in the compartments that induced the crystalline orientations of aragonite tablets had (002), (012), and (102) crystalline plane nets in the early period and some proteins that resulted in the crystalline orientations of aragonite tablets had only (002) crystalline plane nets in the later period. Actually, the experimental results of Fritz et al. [28] demonstrated that aragonites presented multiple crystalline orientations after seven days formation of the nacres and mainly (002) plane net crystalline orientation after fourteen days [28]. Namely, during the formation of the nacres, the crystalline orientations of aragonite tablets tend to be changed from the multiple crystalline plane nets to the (002) crystalline plane net only. Consequently, the multiple crystalline orientations of aragonite tablets and thin nacres implied that the samples only experienced early growth stage of the nacres. Therefore, the samples may be further grown up.

(4) Based on the periodic bond chain (PBC) theory, during the process of crystal growth, there are three kinds of possible crystal planes, namely, flat planes (F), stepped planes $(\mathrm{S})$, and kinked planes $(\mathrm{K})$. The growth velocity of the $\mathrm{K}$ planes is fastest, followed by the $\mathrm{S}$ planes, and the growth velocity of $\mathrm{F}$ planes is the slowest. Consequently, all of the crystal faces are $\mathrm{F}$ planes on the perfect crystal [29]. However, there are many $\mathrm{K}$ planes and/or $\mathrm{S}$ planes on the aragonite crystals in the nacres because the growth of inorganic crystals is controlled by organic matters [6]. Based on the above analyses, the present authors think that the formation processes of the microstructure of the nacres can be divided into three stages. In the early stage, aragonite crystals nucleated and grew up fast. In the middle stage, the aragonite crystals directionally grew up to form the aragonite tablets and microlayers. In the last stage, the aragonite tablets in the aragonite micro-layer mixed perfectly together to form high-quality aragonite layer. In each growth stage, the respective microstructures of the nacres are different.

In the early stage, when the calcium carbonate solution was oversaturated, the precursor particles of aragonite nucleated and grew up fast. Then the aragonite crystals with 
many $\mathrm{K}$ planes and/or $\mathrm{S}$ planes were formed. At the same time, due to the effects of the types and contents of organic matters, the crystalline orientations of aragonite tablets were various. In addition, the effects of organic matters and the inhomogenous crystal growth velocity resulted in the porous fine aragonite crystal aggregates formation (Figures 2(b) and $2(\mathrm{~d}))$.

In the middle stage, the $\mathrm{F}$ planes began to grow and the $\mathrm{K}$ planes grew slowly, and the $\mathrm{S}$ planes were the main growth planes. In this scenario, the aragonite crystal aggregates directionally grew up to form the aragonite tablets and microlayers. The surface of the aragonite tablets and microlayers are rough and few porous, and the edges of the crystals were serrated (Figures 2(a) and 2(b)). Particularly, it was possible for the formation of aragonite crystal domains because the $S$ planes growth could result in forming a "bridge" between the gaps of two adjacent matrices.

In the last stage, the $\mathrm{F}$ planes were the main growth planes and the $\mathrm{K}$ planes and $\mathrm{S}$ planes grew slowly. In this scenario, the aragonite tablets in the aragonite micro-layer mixed perfectly together to form high-quality aragonite layer whose surface was smooth and perfect (Figures 2(f) and 2(i)).

\section{Conclusions}

(1) Based on the pearl luster, size, and surface flaw ratio, the samples can be classified as the high-quality pearls, mediumquality pearls, and inferior-quality pearls. In the nacres of the high and medium-quality pearls, aragonite is the unique inorganic mineralogy. In the nacres of the inferior-quality pearls, aragonite is the main inorganic mineralogy, and there are small amounts of calcites which lower the quality of pearls and result in crystalline polymorphy of calcium carbonate. The formation of calcite in the nacres may be related to the facts that calcium element in the calcium carbonate can be substituted by some trace elements (such as $\mathrm{Fe}, \mathrm{Al}, \mathrm{K})$.

(2) The inorganic matters (such as aragonite) were crystallized and grown up in the compartments formed by the silk and radial organic sheets originating from organic matters secreted by the mantle of mollusks. Due to the effects of the types and contents of organic matters, the crystalline orientations of aragonite tablets was changed from the (002) crystalline plane net whose $\mathrm{C}$-crystal axis was vertical to the stratification plane of the nacres and the (012) and (102) crystalline plane nets whose C-crystal axis were oblique to the stratification plane of the nacres in the early to the (002) crystalline plane net only in the later.

(3) The formation processes of the microstructure of the nacres could be divided into three stages. In the early stage, the precursor particles of aragonite nucleated and grew up fast, then, porous aragonite aggregates consisting of the fine aragonite crystals were formed. In the middle stage, the aragonite crystals directionally grew up to form the aragonite tablets and microlayers. The surface of the aragonite tablets and microlayers are rough and few porous and the edges of the crystals were serrated. In the last stage, the aragonite tablets in the aragonite micro-layer mixed perfectly together to form high-quality aragonite layer whose surface was smooth and perfect.

\section{Acknowledgments}

The authors gratefully acknowledge support for the work by Grant no. 9151027501000088 from the Guangdong Provincial Natural Science Foundation and Grant no. 11lgpy02 from the Fundamental Research Funds for the Central Universities.

\section{References}

[1] A. M. Belcher, P. K. Hansma, G. D. Stucky, and D. E. Morse, "First steps in harnessing the potential of biomineralization as a route to new high-performance composite materials," Acta Materialia, vol. 46, no. 3, pp. 733-736, 1998.

[2] H. Nakahara, M. Kakei, and G. Bevelander, "Electron microscopic and amino acid studies on the outer and inner shell layers of Haliotis rufescens," Venus, vol. 41, no. 1, pp. 33-46, 1982.

[3] N. Watabe, "Crystal growth of calcium carbonate in the invertebrates," Progress In Crystal Growth And Characterization, vol. 4, no. 1-2, pp. 99-147, 1981.

[4] T. E. Schäffer, C. Ionescu-Zanetti, R. Proksch et al., "Does abalone nacre form by heteroepitaxial nucleation or by growth through mineral bridges?" Chemistry of Materials, vol. 9, no. 8, pp. 1731-1740, 1997.

[5] S. Weiner and L. Hood, "Soluble protein of the organic matrix of mollusk shells: a potential template for shell formation," Science, vol. 190, no. 4218, pp. 987-989, 1975.

[6] S. Weiner and L. Addadi, "Acidic macromolecules of mineralized tissues: the controllers of crystal formation," Trends in Biochemical Sciences, vol. 16, no. 7, pp. 252-256, 1991.

[7] G. S. Zhang and X. D. Xie, "Preferential orientation of aragonite crystals in nacre of mollusc shells," Journal of Inorganic Materials, vol. 15, no. 4, p. 768, 2000.

[8] G. Zhang and X. Xie, "Utrastructure and formation theory of nacre shells," Journal of Mineralogy and Petrology, vol. 20, no. 1, pp. 11-16, 2000.

[9] N. Watabe, "Studies on shell formation. XI. Crystal-matrix relationships in the inner layers of mollusk shells," Journal of Ultrasructure Research, vol. 12, no. 3, pp. 351-370, 1965.

[10] S. Manne, C. M. Zaremba, R. Giles et al., "Atomic force microscopy of the nacreous layer in mollusc shells," Proceedings of the Royal Society B, vol. 256, no. 1345, pp. 17-23, 1994.

[11] X. Zhang, W. Wu, and J. Wang, "Dynamic analysis of preferential orientation of aragonite crystals in nacre from mollusk shell," Chinese Science Bulletin, vol. 52, no. 24, pp. 3452-3456, 2007.

[12] F. Song, A. K. Soh, and Y. L. Bai, "Structural and mechanical properties of the organic matrix layers of nacre," Biomaterials, vol. 24, no. 20, pp. 3623-3631, 2003.

[13] E. Zhang, M. Xin, and M. S. Peng, "A study of compositional characteristics of Chinese cultured pearls," Acta Petrolog. Et Mineral, vol. 26, no. 4, pp. 381-386, 2007.

[14] G. S. Zhang and X. H. Li, "Characteristics of FTIR spectra of biogenic aragonite in mollusk shells," Spectroscopy and Spectral Analysis, vol. 25, no. 10, pp. 1588-1590, 2005.

[15] G. S. Zhang and S. L. Ding, "Effect of griding on IR spectra of aragonite," Mineral Resource Geology, vol. 19, no. 110, pp. 429-431, 2005. 
[16] R. W. Gauldie, S. K. Sharma, and E. Volk, "Micro-Raman spectral study of vaterite and aragonite otoliths of the coho salmon, Oncorhynchus kisutch," Comparative Biochemistry and Physiology, vol. 118, no. 3, pp. 753-757, 1997.

[17] Y. L. Hao, Situ Raman Spectra of Freshwater Cultured Pearls and the Phase Transformation of Its Inorganic Cystals, Guangxi University, Nanning, China, 2005.

[18] S. M. dePaula, M. F. G. Huila, K. Araki, and H. E. Toma, "Confocal Raman and electronic microscopy studies on the topotactic conversion of calcium carbonate from Pomacea lineate shells into hydroxyapatite bioceramic materials in phosphate media," Micron, vol. 41, no. 8, pp. 983-989, 2010.

[19] S. Raz, S. Weiner, and L. Addadi, "Formation of highmagnesian calcites via an amorphous precursor phase: possible biological implications," Advanced Materials, vol. 12, no. 1, pp. 38-42, 2000.

[20] G. Falini, S. Fermani, M. Gazzano, and A. Ripamonti, "Polymorphism and architectural crystal assembly of calcium carbonate in biologically inspired polymeric matrices," Journal of the Chemical Society, Dalton Transactions, no. 21, pp. 39833987,2000

[21] J. J. J. M. Donners, B. R. Heywood, E. W. Meijer, R. J. M. Nolte, and N. A. J. M. Sommerdijk, "Control over calcium carbonate phase formation by dendrimer/surfactant Templates," Chemistry, vol. 8, no. 11, pp. 2561-2567, 2002.

[22] A. Sugawara and T. Kato, "Aragonite $\mathrm{CaCO}_{3}$ thin-film formation by cooperation of $\mathrm{Mg}^{2+}$ and organic polymer matrices," Chemical Communications, no. 6, pp. 487-488, 2000.

[23] J. C. Marxen and W. Becker, "Calcium binding constituents of the organic shell matrix from the freshwater snail Biomphalaria glabrata," Comparative Biochemistry and Physiology, vol. 127, no. 2, pp. 235-242, 2000.

[24] V. T. C. Chang, R. J. P. Williams, A. Makishima, N. S. Belshawl, and R. K. O'Nions, 'Mg and $\mathrm{Ca}$ isotope fractionation during $\mathrm{CaCO}_{3}$ biomineralisation," Biochemical and Biophysical Research Communications, vol. 323, no. 1, pp. 79-85, 2004.

[25] B. Pokroy, J. P. Quintana, E. N. Caspi, A. Berner, and E. Zolotoyabko, "Anisotropic lattice distortions in biogenic aragonite," Nature Materials, vol. 3, no. 12, pp. 900-902, 2004.

[26] S. Weiner, Y. Levi-Kalisman, S. Raz, and L. Addadi, "Biologically formed amorphous calcium carbonate," Connective Tissue Research, vol. 44, no. 1, pp. 214-218, 2003.

[27] M. Suzuki, K. Saruwatari, T. Kogure et al., "An acidic matrix protein, Pif, is a key macromolecule for nacre formation," Science, vol. 325, no. 5946, pp. 1388-1390, 2009.

[28] M. Fritz, A. M. Belcher, M. Radmacher et al., "Flat pearls from biofabrication of organized composites on inorganic substrates," Nature, vol. 371, no. 6492, pp. 49-51, 1994.

[29] Y. F. Ma, Y. H. Gao, D. N. Ren, and Q. L. Feng, "The effect of soluble matrix proteins from aragonite pearls on the crystallization of $\mathrm{CaCO}_{3}$," Acta Petrol. Et Mineral, vol. 28, no. 6, pp. 605-610, 2009. 


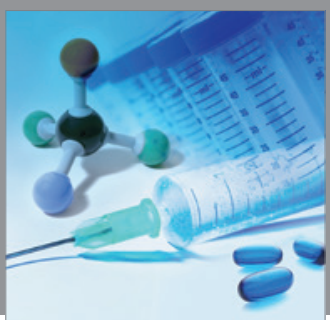

International Journal of

Medicinal Chemistry

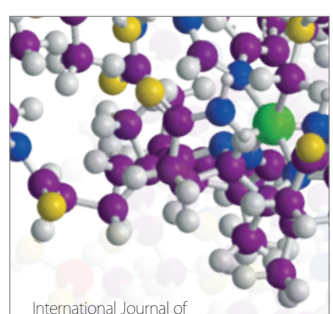

Carbohydrate Chemistry

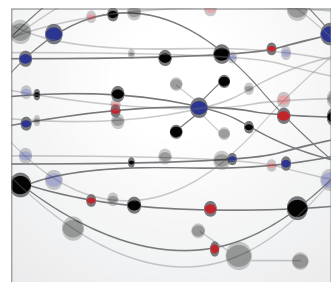

The Scientific World Journal
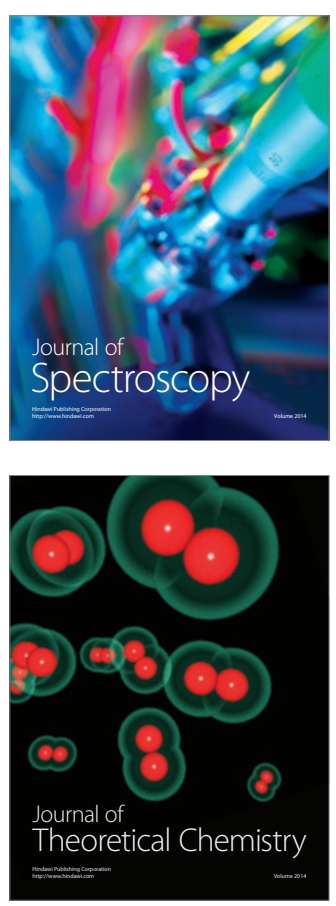
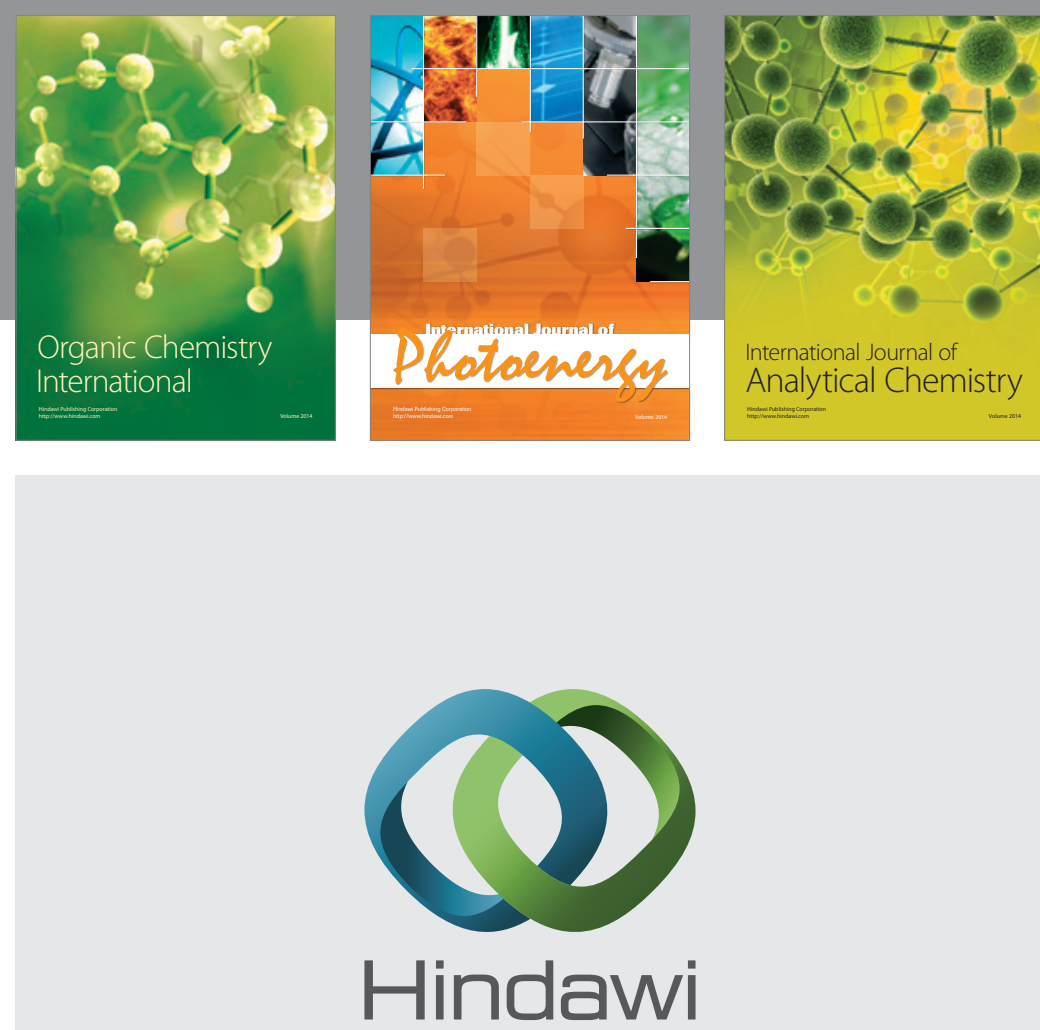

Submit your manuscripts at

http://www.hindawi.com
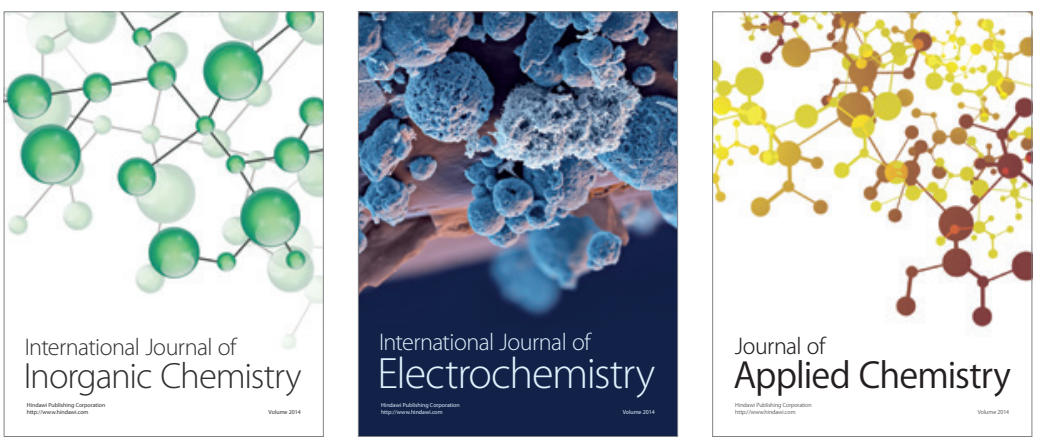

Journal of

Applied Chemistry
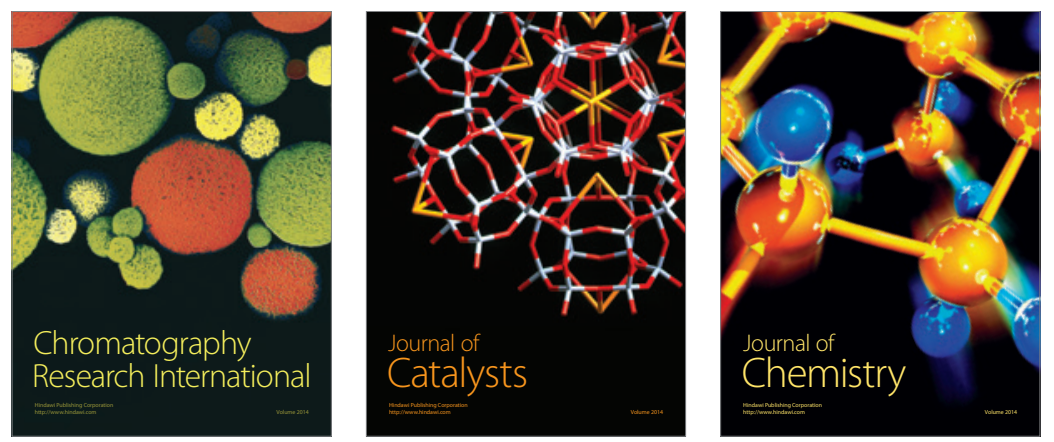
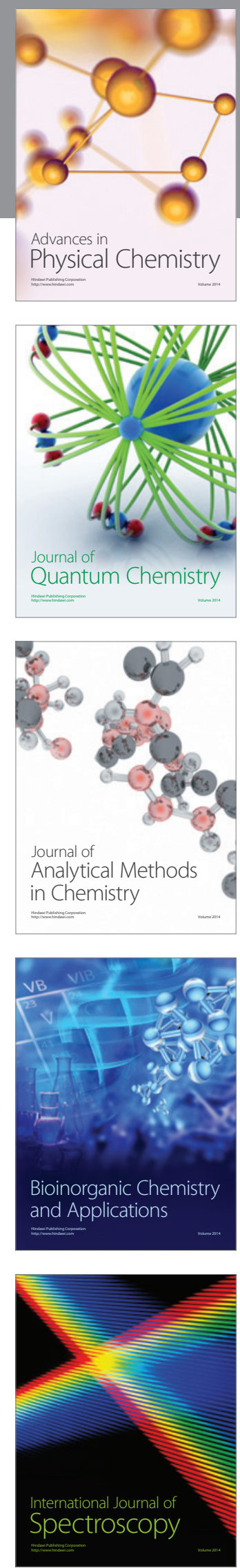